\title{
Prognosis of patients with diffuse large B cell lymphoma not reaching complete response or relapsing after frontline chemotherapy or immunochemotherapy
}

\author{
Jordina Rovira • Alexandra Valera - Lluis Colomo • Xavier Setoain • Sonia Rodríguez • \\ Alejandra Martínez-Trillos • Eva Giné • Ivan Dlouhy • Laura Magnano • Anna Gaya • \\ Daniel Martínez • Antonio Martínez • Elías Campo • Armando López-Guillermo
}

Received: 18 August 2014 / Accepted: 1 December 2014 / Published online: 14 December 2014

(C) The Author(s) 2014. This article is published with open access at Springerlink.com

\begin{abstract}
A retrospective study was performed to assess the outcome of patients with diffuse large B cell lymphoma (DLBCL) who did not achieve complete response or who relapsed before and after the use of rituximab. Clinical features and outcome of $816(425 \mathrm{M} / 391 \mathrm{~F}$; median age 63 years) patients diagnosed from 1991 to 2001 (pre-rituximab era, $N=348$ ) and from 2002 to 2012 (rituximab era, $N=468$ ) in a single institution were evaluated. Five hundred fiftythree patients achieved complete remission (CR), 57 partial response (PR), and 206 were refractory with a median overall survival of $15,1.5$, and 0.4 years, respectively. Patients receiving rituximab had lower risk of refractoriness or relapse. In primarily refractory and PR patients, there was not a difference in survival depending on whether patients received or not rituximab-containing frontline treatment. Early death rate was $11 \%$, including $3.6 \%$ due to infectious complications. Rituximab did not modify these figures. In the relapse setting, 5 -year survival from relapse was $25 \%$ for patients who never received rituximab, $54 \%$ for those who received rituximab only at relapse, and $48 \%$ for those treated with immunochemotherapy
\end{abstract}

J. Rovira $(\triangle) \cdot$ A. Martínez-Trillos $\cdot$ E. Giné $\cdot$ I. Dlouhy $•$

L. Magnano · A. Gaya $\cdot$ A. López-Guillermo

Hematology Department, Hospital Clínic, IDIBAPS,

C/. Villarroel, 170, 08036 Barcelona, Spain

e-mail: jorovira@clinic.ub.es

A. Valera $\cdot$ L. Colomo $\cdot$ D. Martínez $\cdot$ A. Martínez $\cdot$ E. Campo

Pathology Department, Hospital Clínic, IDIBAPS,

University of Barcelona, Barcelona, Spain

X. Setoain

Nuclear Medicine Department, Hospital Clínic, Barcelona, Spain

S. Rodríguez

Radiology Department, Hospital Clínic, Barcelona, Spain both as frontline and at relapse. In conclusion, relapsed/ refractory patients with DLBCL show poor prognosis despite the use of frontline immunochemotherapy. New therapeutic approaches are needed in this group of patients.

Keywords Relapse/refractory · DLBCL .

Immunochemotherapy

\section{Introduction}

Diffuse large B cell lymphoma (DLBCL) is the commonest subtype of non-Hodgkin's lymphoma accounting for approximately $30-50 \%$ of cases [1]. DLBCL shows an aggressive behavior with a median survival of less than 1 year in untreated patients. Since the 1970s, the CHOP regimen (cyclophosphamide, adriamycin, vincristine, and prednisone) has been the standard treatment with $50 \%$ of complete remissions (CR) and $30-40 \%$ of long survivors [2]. Prognosis of DLBCL has considerably improved during the last decade, mainly due to the addition of rituximab, the first approved antiCD20 monoclonal antibody, to chemotherapy (CT), known as immunochemotherapy. The addition of rituximab to firstline treatment improved CRs by 15-20\%, 5-year event-free survival (EFS) from 29 to $47 \%$ in patients 60 to 80 years old and 3-year EFS or progression-free survival (PFS) from 59 to $79 \%$ in patients aged 18 to 60 [3-9]. More importantly, immunochemotherapy significantly improved overall survival (OS) [3-9]. Therefore, rituximabCT (R-CT) became the new standard of care by 2002 . However, despite these notable advancements, there is still a considerable proportion of patients that are primarily refractory or experience short-term relapses impairing their possibilities of survival. For patients with chemosensitive 
disease, the standard treatment for relapsed/refractory DLBCL cases is salvage CT followed by autologous stem cell transplantation (ASCT) [10]. Patients who progress while receiving frontline therapy or those relapsing very early (the so-called primary chemorefractory patients) are less likely to respond to salvage treatment and therefore, have a clearly inferior PFS and OS than late relapses [11, 12]. After the PARMA study, several retrospective analyses have supported the role of ASCT in primarily chemorefractory cases $[13,14]$. Typical salvage CT regimens are platinum based plus high-dose cytarabine, ifosfamide or gemcitabine. Currently, the use of immunochemotherapy at relapse is generalized, although the evidence to support this fact, particularly in R-CT refractory patients, is scarce. Moreover, it has been suggested that the prognosis of relapsed/refractory patients is worse nowadays than before rituximab $[15,16]$. Whether the outcome of DLBCL patients not reaching CR or who eventually relapse has changed in the last decade with the use of frontline immunochemotherapy is still an open question.

In this setting, the aim of the present study was to assess the clinical features, response to salvage treatment, PFS and OS of patients with relapsed/refractory DLBCL before and after the addition of rituximab to firstline therapy in a single institution.

\section{Patients and methods}

\section{Patients}

Nine hundred eighty-three patients were consecutively diagnosed with DLBCL according to the WHO classification [17], in a single institution between 1991 and 2012. The only criterion to include patients was the availability of histological material. Patients with post-transplant lymphoproliferative disorders $(N=9)$, immunodeficiency-associated $(N=95)$, central nervous system $(N=39)$, primary effusion $(N=1)$, and primarily mediastinal lymphomas $(N=37)$ were excluded. Thus, 816 patients were the subject of the present study. Patients were divided in two groups: 348 patients (42.6\%) diagnosed during the pre-rituximab era (1991-2001) (pre-R) and 468 patients $(57.4 \%)$ during the rituximab period (20022012) (R). The main characteristics of patients are described in Table 1. The median age of patients was 63 years (range 14-94) and the male/female distribution 425 (52\%)/391 (48\%). Most variables showed no significant differences through time, but during the last decade, the median age was higher and the proportion of extranodal involvement lower. The first cohort of patients received chemotherapy (CT) and the second one immunochemotherapy (R-CT) unless intolerance or toxicity $(N=3)$. The frontline regimens slightly varied over time and could be summarized as curative intention (CHOP or CHOP-like) or palliative intention (COP) depending on the use or not of adriamycin. The proportion of patients treated with curative intention was higher in the second cohort than that in the first one ( $85 \mathrm{vs} 78 \%$, respectively; $P=0.01$ ).

The study was performed according to the guidelines of the Ethic Committee of the Hospital Clínic of Barcelona. Informed consent to use the clinical data was obtained in accordance with the Declaration of Helsinki.

\section{Staging and treatment}

The following variables were recorded and analyzed: (i) demographic data, performance status according to the Eastern Cooperative Oncology Group (ECOG) scale, presence of B symptoms (fever, night sweats, weight loss), and bulky disease (defined as a tumor diameter $>7 \mathrm{~cm}$ ); (ii) hematological and biochemical parameters: blood cell counts, hemoglobin, serum lactate dehydrogenase (LDH), and $\beta_{2}$-microglobulin $\left(\beta_{2} \mathrm{~m}\right)$ levels; (iii) tumor extension data: nodal and extranodal involvement, number of extranodal involved sites, palpable splenomegaly or hepatomegaly, bone marrow infiltration and Ann Arbor Stage; (iv) the International Prognostic Index (IPI) [18]; (v) treatment; response to treatment, relapse, cause of death; (vi) appearance of secondary neoplasias.

\section{Response to therapy and outcome}

The criteria to assess response were those of Cheson 1999, based on CT scan. Thus, CR was defined as the total disappearance of tumor masses and disease-related symptoms as well as the normalization of the initial abnormal test for at least 1 month. $\mathrm{CR} /$ unconfirmed (CRu) included a residual lymph node mass greater than $1.5 \mathrm{~cm}$ that regressed more than $75 \%$ and individual nodes that were previously confluent that regressed more than $75 \%$. Partial response (PR) was considered when tumor mass or organ infiltration decreased by at least $50 \%$ along with the disappearance of disease-related symptoms. $\mathrm{CR}$ and $\mathrm{CRu}$ patients were analyzed together. In addition, since 2005 when PET scan became available in our institution, this procedure was used to assess response, and therefore, patients with residual masses were considered in PR when PET scan was positive and in CR when PET scan was negative [19]. Patients not included in these categories and early deaths were considered nonresponders. Early death was defined as those patients dying within the 4 months after diagnosis. Disease relapse or progression was defined as the appearance of new symptoms or signs of the disease as demonstrated by lymph node biopsy or other appropriate studies. OS and PFS were calculated according to standard definitions [19].

Statistical methods

Differences among the subgroups of patients were assessed by using the Chi-square test (two-tailed), the Student's $t$ test, or 
Table 1 Clinical characteristics at diagnosis of 816 patients with diffuse large B cell lymphoma
ECOG Eastern Cooperative Oncology Group, $L D H$ lactate dehydrogenase, $\beta_{2} m \beta_{2^{-}}$ microglobulin, $B M$ bone marrow $* P<0.05$

\begin{tabular}{|c|c|c|c|}
\hline & $\begin{array}{l}\text { All } \\
N(\%)\end{array}$ & $\begin{array}{l}1991-2001 \\
N(\%)\end{array}$ & $\begin{array}{l}2002-2012 \\
N(\%)\end{array}$ \\
\hline Patients & $816(100)$ & $348(43)$ & $468(57)$ \\
\hline \multicolumn{4}{|l|}{ Gender } \\
\hline Male & $425(52)$ & $167(48)$ & $258(55)^{*}$ \\
\hline Female & $391(48)$ & $181(52)$ & $210(45)$ \\
\hline \multicolumn{4}{|l|}{ Age } \\
\hline$\leq 60$ & $364(45)$ & $174(50)$ & $190(41)^{*}$ \\
\hline$>60$ & $452(55)$ & $174(50)$ & $278(59)$ \\
\hline B symptoms & $319(40)$ & $132(38)$ & $187(41)$ \\
\hline \multicolumn{4}{|l|}{ ECOG performance status } \\
\hline$<2$ & $465(59)$ & $191(55)$ & $274(62)$ \\
\hline$\geq 2$ & $319(41)$ & $153(44)$ & $166(38)$ \\
\hline Bulky disease $(>7 \mathrm{~cm})$ & $226(29)$ & $102(30)$ & $124(29)$ \\
\hline Primarily extranodal & $290(35)$ & $140(40)$ & $150(32)^{*}$ \\
\hline Extranodal involvement & $525(64)$ & $242(70)$ & $283(60)^{*}$ \\
\hline BM involvement & $126(16)$ & $65(19)$ & $61(13)^{*}$ \\
\hline \multicolumn{4}{|l|}{ Ann Arbor stage } \\
\hline I-II & $380(47)$ & $175(51)$ & $205(45)$ \\
\hline III-IV & $426(53)$ & $171(49)$ & $255(55)$ \\
\hline High serum LDH & $395(52)$ & $181(55)$ & $214(50)$ \\
\hline High serum $\beta 2 \mathrm{~m}$ & $280(46)$ & $103(45)$ & $177(47)$ \\
\hline \multicolumn{4}{|l|}{ IPI risk group } \\
\hline Low & $271(35)$ & $120(36)$ & $151(34)$ \\
\hline Low intermediate/high intermediate & $326(42)$ & $127(38)$ & $199(45)$ \\
\hline High & $179(23)$ & $83(25)$ & $96(21)$ \\
\hline
\end{tabular}

nonparametric tests when necessary. The actuarial survival analysis was performed by the Kaplan and Meier method and differences assessed by the log-rank test. To evaluate the prognostic impact of different variables in response to salvage therapy, PFS, and OS, multivariate analyses were performed with the stepwise proportional hazards model (Cox model) [20]. $P$ values $<0.05$ were considered statistically significant.

\section{Results}

Response to frontline treatment and outcome

After therapy, 553 patients (68\%) achieved CR, 57 PR (7\%), and 206 did not respond (25\%). As expected, the CR rate was higher in patients treated with R-CT compared to those receiving CT alone ( 71 vs $64 \%$, respectively; $P=0.036$ ) and particularly in patients treated with curative intention (77 vs $68 \%$, respectively; $P=0.009$ ). In addition, the proportion of primary refractory cases was significantly lower in the R-CT subgroup (29 vs $23 \%$, respectively;
$P=0.05)$, specifically in patients treated with curative intention (24 vs $15 \%$, respectively; $P=0.009$ ). Those patients $(N=263)$ who did not achieve CR were older and more often had poor ECOG performance status, B symptoms, advanced Ann Arbor stage, bulky disease, extranodal involvement, bone marrow and CNS infiltration, high serum LDH and $\beta_{2} \mathrm{~m}$, high-risk IPI and received palliative approach as compared with those achieving CR $(P \leq 0.008$ in all cases).

Median follow-up for surviving patients was 6.5 years (range 0.02-23.2). One hundred fifty out of 553 patients in CR eventually relapsed. Five-year PFS was $45.5 \%$ (95\% CI 44.4-46.6\%), with significant differences between the pre-R and R period (5-year PFS 39 vs $51 \%$, respectively; $P=0.002$ ) (Table 2). This difference was also observed in patients treated with curative intention (5-year PFS 45 vs $57 \%$, respectively; $P=0.002)$. Four hundred and nineteen patients died during the follow-up with a 5-year OS of $54 \%$ (95 \% CI 50.2-57.2\%), with a significant difference between pre-R and $\mathrm{R}$ eras (5-year OS 48 vs $59 \%$, respectively; $P=0.004$ ). Once again, the difference was maintained in patients treated with curative intention (5-year OS 55 vs $65 \%$, respectively; $P=0.006$ ). A multivariate analysis showed that in a model of 582 patients, 
Table 2 Outcome of 816 patients with diffuse large B cell lymphoma

\begin{tabular}{|c|c|c|c|c|}
\hline & $\begin{array}{l}\text { Whole series } \\
N=816\end{array}$ & $\begin{array}{l}1991-2001 \\
N=348\end{array}$ & $\begin{array}{l}2002-2012 \\
N=468\end{array}$ & $P$ value \\
\hline Treatment response, $N(\%)$ & & & & 0.036 \\
\hline $\mathrm{CR}$ & $553(68)$ & $222(64)$ & $331(71)$ & \\
\hline PR & $57(7)$ & $26(7)$ & $31(7)$ & \\
\hline No response & $206(25)$ & $100(29)$ & $106(23)$ & \\
\hline PFS at 5 years $(\%)$ & 45.5 & 39 & 51 & 0.002 \\
\hline OS at 5 years $(\%)$ & 53.7 & 48 & 59 & 0.004 \\
\hline Relapse after CR, $N(\%)$ & $150(27)$ & $81(36)$ & $69(21)$ & $<0.0001$ \\
\hline Survival from relapse at 5 years $(\%)$ & 29 & 29 & 35 & NS \\
\hline Relapse $\leq 2$ years from CR (\%) & 20 & 21 & 19 & NS \\
\hline Relapse >2 years from CR (\%) & 46 & 44 & 48 & NS \\
\hline
\end{tabular}

$C R$ complete response, $P R$ partial response, $P F S$ progression-free survival, $O S$ overall survival

IPI $(P<0.0001$, HR 1.9, $95 \%$ CI 1.7-2.3), use of rituximab $(P<0.0001$, HR 0.5, $95 \%$ CI $0.4-0.6)$ and bulky disease $(P=0.048$, HR $1.3,95 \%$ CI $1.0-1.6)$ were the most important variables affecting OS. PFS and OS curves of the whole series and of those patients treated with curative intention are shown in Fig. 1. The cause of death was lymphoma in $83 \%$ of cases which was similar in both groups. Twenty-seven second neoplasias (3.4\%) were detected during the follow-up with no significant differences between the two subgroups.
Salvage treatment in primary chemorefractory patients

Ninety-two out of 206 patients (45\%) who did not reach a response died within 4 months from diagnosis, including 10 patients who were never treated. These early death rates in patients receiving CT or R-CT were 35/348 (10\%) versus 57/ 468 (12\%), respectively. Infectious complications were the ultimate cause of death in 30 cases (3.2 vs $4 \%$ in pre-R and $R$ era, respectively), irrespective of the possible response of the disease. One hundred fourteen patients surviving more than
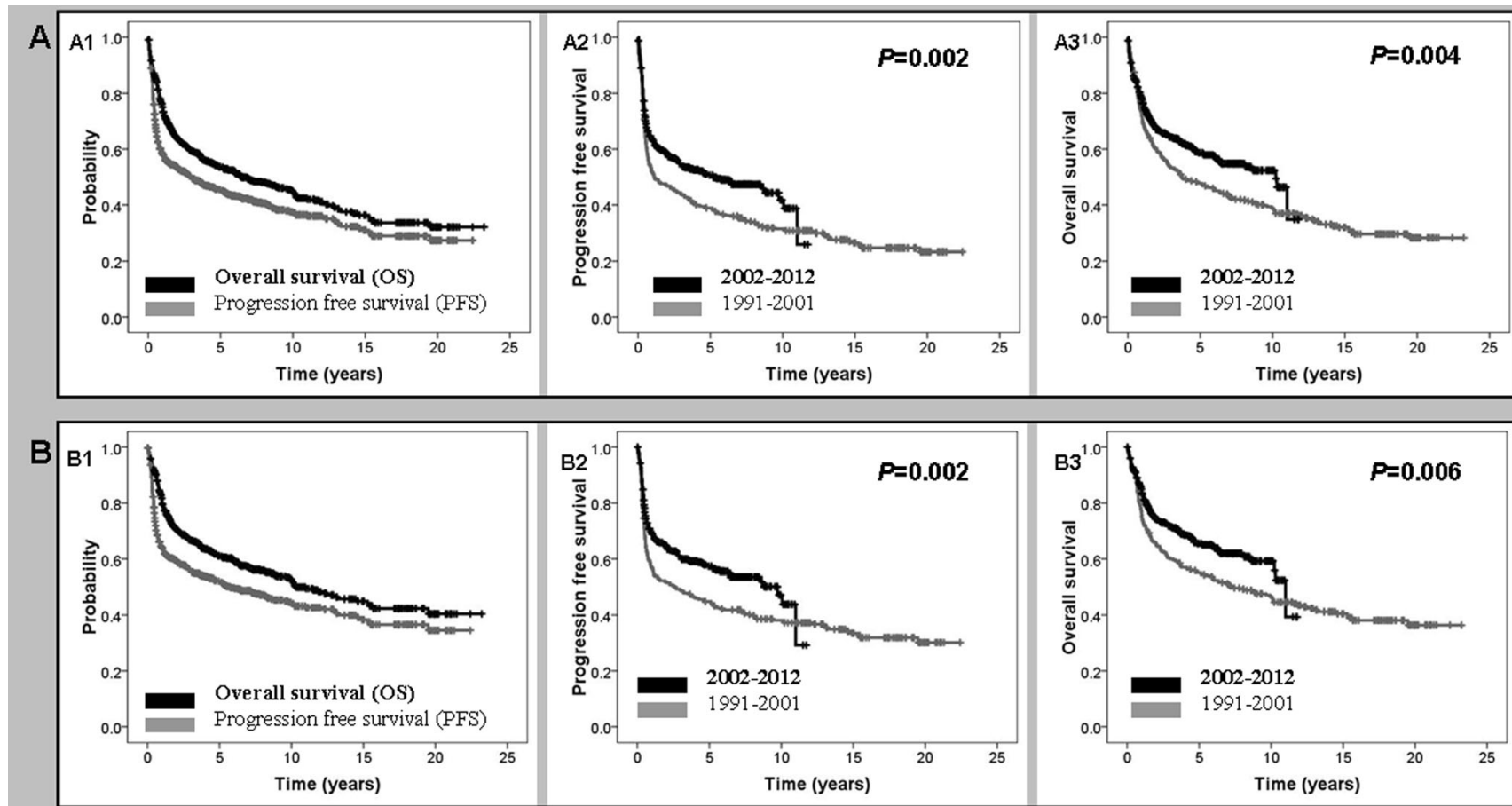

Fig. 1 Outcome of the whole series of patients with diffuse large B cell lymphoma (a) and of those treated with curative intention (b). Overall survival (OS) and progression-free survival (PFS) of the subgroups (a1, b1). PFS according to the year of diagnosis (before and after December 2001) (a2, b2). OS according to the year of diagnosis (before and after December 2001) (a3, b3) 
4 months were primary refractory to treatment; the median OS of this group was 0.75 years (Fig. 2a). Sixty-one of these patients (31 pre-R; $30 \mathrm{R}$ ) received only palliative measures mainly due to age and/or poor ECOG performance status, and all of them died between 4 and 44 months from diagnosis. Salvage treatment was administered to 53 patients (34 and 19 in the pre- $\mathrm{R}$ and $\mathrm{R}$ era, respectively). In the pre-R era, only one patient achieved CR (3\%) and three PR $(9 \%)$ whereas in the $\mathrm{R}$ era, three patients achieved CR (16\%) and five PR (26\%) $(P=0.027)$. One patient in $\mathrm{PR}$ in the pre-R era received allogeneic stem cell transplantation (Allo-SCT). This patient died in CR at 7.2 years after transplant due to esophagus carcinoma. In the R era, three patients received an ASCT and one an Allo-SCT. The only patient in the pre-R era who achieved CR died due to a stroke 14 years from the assessment of response. Three patients in the $\mathrm{R}$ era were long survivors. Overall, the median survival after salvage therapy of those patients treated with curative intention was 0.46 years $(0.39$ years in pre- $R$ era vs 0.64 years in $\mathrm{R}$ era; $P=0.044$ ) (Fig. $2 \mathrm{~b}$ ). Responses and survival after salvage treatment are summarized in Table 3.

\section{Salvage treatment in patients in PR}

Fifty-seven patients were in PR after induction therapy, 26/348 (7.5 \%) treated with CT, and 31/468 (7\%) with R-CT. When restricted to curative treatment, the figures were $22 / 271(8.1 \%)$ and $29 / 397(7.3 \%)$. Fifteen of the 57 patients (6 pre-R; 9 R) received only palliative measures, whereas 42 (20 pre-R; $22 \mathrm{R})$ received ESHAP salvage therapy (etoposide, methylprednisolone, high-dose cytarabine, and cisplatin). Only two patients in the pre-R era received rituximab as part of salvage therapy, whereas all the cases in the $\mathrm{R}$ era were treated with R-ESHAP. CR rates were $25 \%$ ( 5 out of 20 ) and $50 \%$ (11 out of 22 ) in the pre-R and R era, respectively $(P=0.09)$. ASCT was performed in four
pre-R and nine R patients. Long survival was $11.5 \%(N=3)$ and $35 \%(N=11)$, respectively $(P=0.03)$. Overall, the median survival after salvage treatment was 1 year, with a 5 -year survival from rescue therapy of 19 vs $32 \%$ for patients in the pre-R and $\mathrm{R}$ eras (Fig. 3a). Regarding patients treated with curative intention, the 5 -year survival after salvage treatment was 26 vs $38 \%$ (Fig. 3b and Table 3). Among four patients who underwent ASCT in the pre-R era, two achieved CR and two died early after ASCT due to cardiogenic shock and infection. One patient in CR died shortly afterward of disease progression. In the $\mathrm{R}$ era, six out of nine patients achieved $\mathrm{CR}$, three died of disease progression, and two relapsed and were treated with a reduced intensity Allo-SCT. One of them died due to infectious complication, and the other patient is alive after 10 years.

\section{Salvage treatment in relapsed patients}

One hundred and fifty out of 553 patients $(27.1 \%)$ in CR eventually relapsed (Table 2). Such proportion was 36 and $21 \%$ during the pre- $\mathrm{R}$ and $\mathrm{R}$ era, respectively $(P<0.0001)$. In the 70 patients in whom a new biopsy was performed, the histology at relapse corresponded to DLBCL in $95 \%$ of cases and to a downgrade relapse in $5 \%$ (all follicular lymphoma). Relapse was primarily extranodal in 58 cases. No differences in the localization relapse or histology were observed between the pre- $\mathrm{R}$ and $\mathrm{R}$ eras. During the last decade, the CR duration (time from $\mathrm{CR}$ achievement to relapse) has been significantly longer than that in the pre-R era (median time 0.75 vs 1.5 years; $P=0.027$ ). Finally, more relapses occurred in older patients ( $>70$ years), namely, $17 \%$ pre-R vs $39 \% \mathrm{R}$ era $(P=0.003)$.

Salvage treatment consisted of CT or R-CT followed by ASCT for those patients $<65-70$ years of age, good performance status and achieving PR or CR after rescue. This
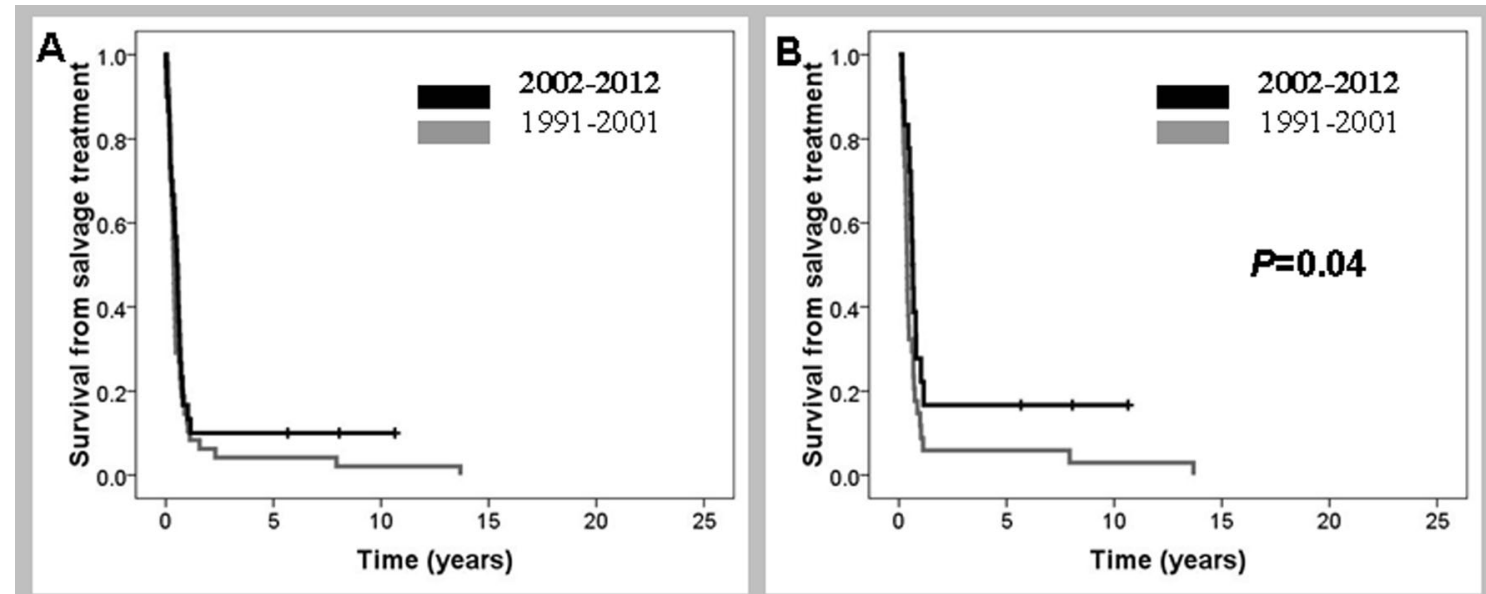

Fig. 2 Survival from salvage treatment of frontline chemorefractory patients diagnosed before or after December 2001. a All patients; b only patients with curative intention to salvage treatment 
Table 3 Outcome of 668 patients with diffuse large B cell lymphoma with chemorefractory disease, partial remission (PR), or relapse, treated with curative intention

\begin{tabular}{|c|c|c|c|c|c|c|}
\hline & \multicolumn{2}{|c|}{ Primary chemorefractory } & \multicolumn{2}{|l|}{$\mathrm{PR}$} & \multicolumn{2}{|l|}{ Relapsed } \\
\hline & $\begin{array}{l}1991-2001 \\
N=34\end{array}$ & $\begin{array}{l}2002-2012 \\
N=19\end{array}$ & $\begin{array}{l}1991-2001 \\
N=18\end{array}$ & $\begin{array}{l}2002-2012 \\
N=22\end{array}$ & $\begin{array}{l}1991-2001 \\
N=57\end{array}$ & $\begin{array}{l}2002-2012 \\
N=34\end{array}$ \\
\hline Response to salvage treatment, $N(\%)$ & $1(3)$ & $3(17)$ & $5(28)$ & $11(50)$ & $29(51)$ & $20(59)$ \\
\hline $\mathrm{CR}$ & $3(9)$ & $5(26)$ & $4(22)$ & $3(14)$ & $6(10)$ & $5(15)$ \\
\hline $\begin{array}{l}\mathrm{PR} \\
\text { No response }\end{array}$ & $30(88)$ & $11(58)$ & $9(50)$ & $8(36)$ & $22(39)$ & $9(26)$ \\
\hline $\operatorname{ASCT}(\%)$ & $3(9)$ & $4(21)$ & $4(22)$ & $9(41)$ & $21(37)$ & $9(26)$ \\
\hline 5-year survival from progression (\%) & 6 & $15^{*}$ & 26 & 38 & 33 & 42 \\
\hline
\end{tabular}

$C R$ complete response, $P R$ partial response, $A S C T$ autologous stem cell transplantation

$* P<0.05$

intensive approach was performed in $82 \%$ of pre-R patients and $56 \%$ of $R$ patients. This difference was because the second group was older. On the other hand, 11 of 76 patients initially treated only with CT received rituximab at relapse, whereas all patients treated in the $\mathrm{R}$ era received rituximab at relapse.

CR rates after salvage therapy were 42 versus $39 \%$ in the pre- $\mathrm{R}$ and $\mathrm{R}$ era, respectively. In those patients treated with curative intention, CR rates were 51 versus $59 \%$ (Table 3). Median survival from relapse (SFR) was 1.12 years, with a $29 \%$ pre-R versus $28 \%$ R 5 -year SFR in pre-R and R eras (Fig. 4a). Median SFR of patients treated with curative intention was 2 years, whereas 5 -year SFR was 33 versus $42 \%$ in the pre-R and R eras, respectively (Fig. 4b). Variables at relapse predicting shorter SFR were older age (5-year SFR of 39 versus $22 \%$ for patients $<60$ and $\geq 60$ years, respectively, $P=0.04$ ) and CNS relapse (5-year SFR of 37 and $18 \%$ patients with or without CNS involvement, respectively, $P=0.02$ ). A trend to shorter SFR was observed in those patients relapsing during the first 2 years after CR achievement (5-year SFR of 20 versus $46 \%$ for patients relapsing $\leq 2$ years or later, $P=0.07)$. SFR is shown in Fig. 5 according to the use of rituximab in front line and at relapse. Five-year SFR was 25,54 , and $48 \%$ for patients who never received rituximab, those who received the drug only at relapse and those treated with rituximab both in front line and rescue regimen, respectively $(P=0.007)$. Such differences were maintained in patients treated with curative intention (Fig. 5b). A multivariate analysis showed that in a model of 69 patients, age at relapse $>70$ years $(P=0.023$, HR $2.1,95 \% \mathrm{CI} 1.1-3.9)$ and CNS involvement $(P=0.04$, HR 2.1, $95 \%$ CI 1.0-4.4) were the most important variables to predict SFR.

\section{Discussion}

Since the 1970s, the treatment of patients with DLBCL has been based on CT. The addition of rituximab to CT dramatically improved the outcome of these patients, as demonstrated
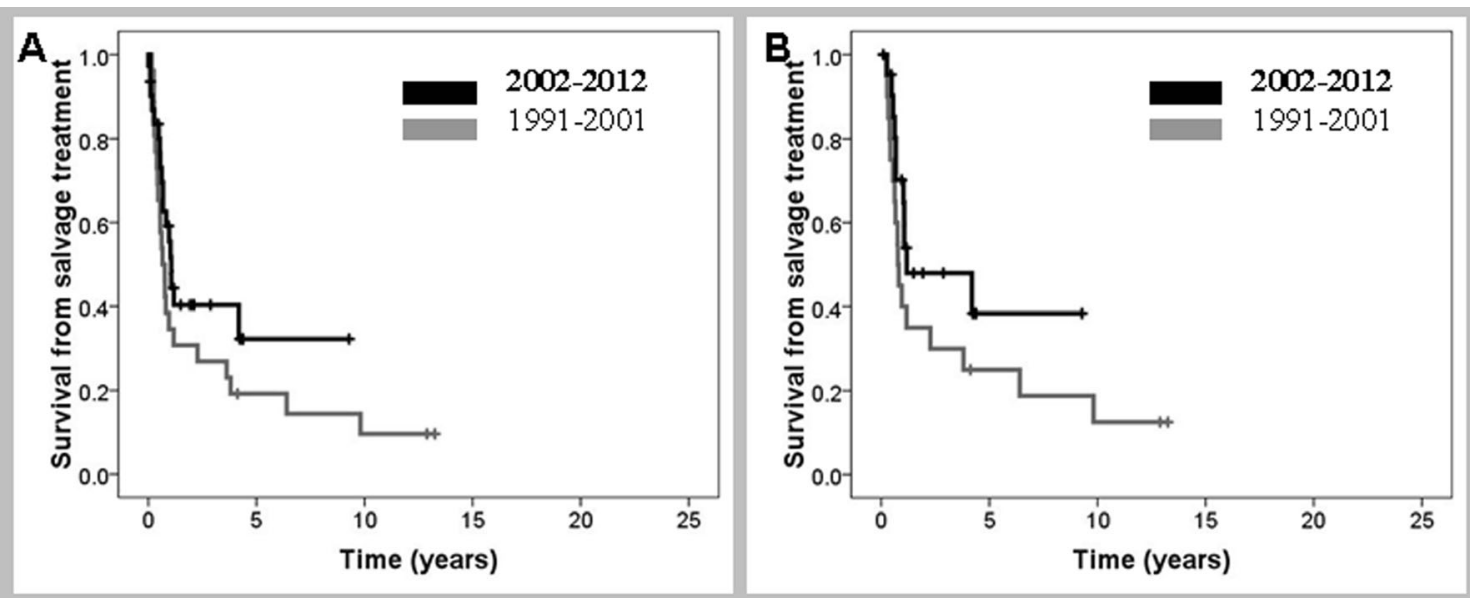

Fig. 3 Survival from salvage treatment of patients in partial remission after frontline therapy diagnosed before or after December 2001 . a All patients; b only patients with curative intention to salvage treatment 

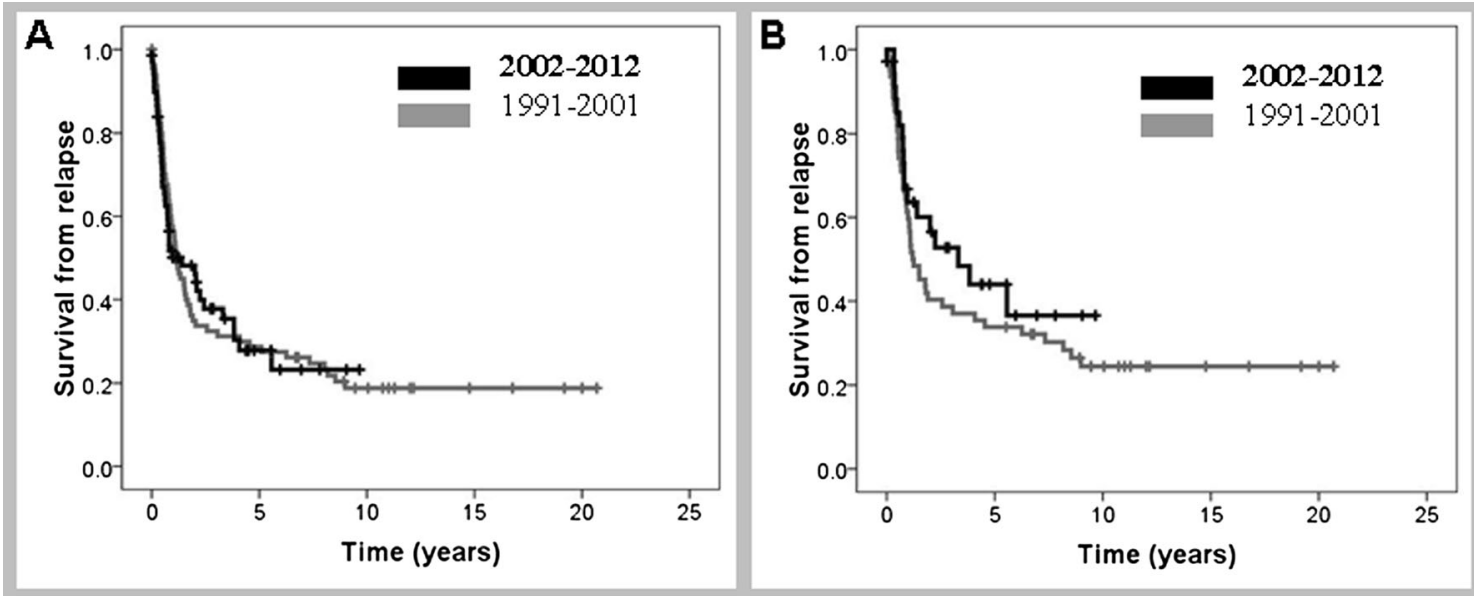

Fig. 4 Survival from relapse diagnosed before or after December 2001. a All patients; $\mathbf{b}$ only patients with curative intention to salvage treatment

in clinical trials and in retrospective population-based studies [3-9]. Thus, immunochemotherapy is currently the gold standard treatment for any CD20-positive DLBCL [3]. Despite this advance, a considerable number of patients will experience early failure, partial response, or relapse after initial rituximab-CT (R-CT). Nowadays, the outcome of relapsed/ refractory $(R / R)$ patients is still poor. Some evidence suggests that patients treated with $\mathrm{R}-\mathrm{CT}$ could be more resistant to salvage therapy than before the use of rituximab. In this setting and in order to highlight the challenges faced between the prerituximab era and the current immunochemotherapy, the aim of our study was to evaluate the characteristics and outcome of those patients with R/R DLBCL after frontline treatment in a single institution. Published data concerning salvage treatment is most often based on highly selected series of patients in whom intensive treatment is possible [4, 6, 10-12, 21-25]. Such cases are not representative of the entire population of patients who fail to initial therapy. An analysis of an unselected series of nonresponders, as herein done, can offer a more realistic view of the efficacy of salvage treatment and the real outcome in the general population.

Patients dying during induction treatment constitute a particular category of nonresponders. In the present study, 92 patients $(11 \%$ of the overall series and $45 \%$ of nonresponders) died during the induction period. It is often difficult to distinguish between toxic death and disease progression as causes of death. In our series, $3.7 \%$ of patients died due to infectious complications. No differences were observed between patients receiving CT or R-CT. This mortality rate is similar to that reported in the literature [3, 5, 26]. After excluding early deaths, primary refractory patients were considered as a different category which accounted for $14 \%$ of the present series. More than half of them received only palliative measures mainly due to older age and/or poor performance status. All of them died within the next 3 years. Only $9 \%$ of rescued patients achieved CR, although this proportion has improved in the last decade. These data apparently contrast with those reported in the

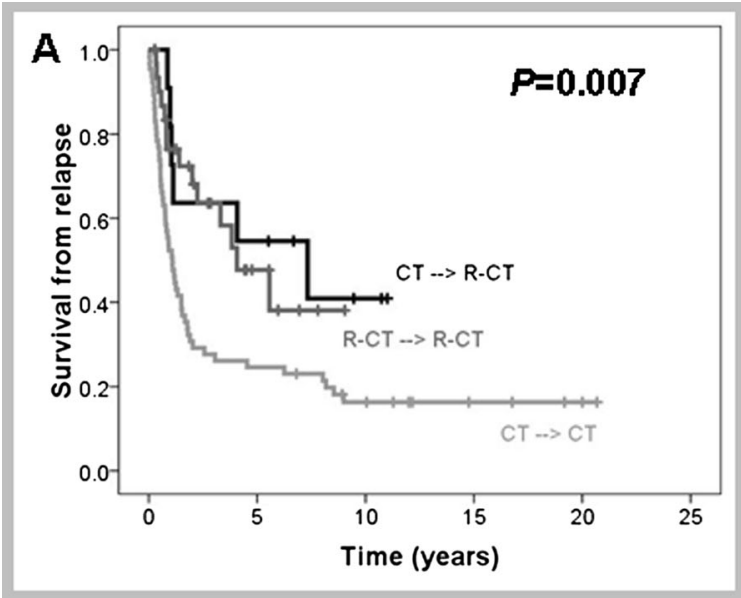

Fig. 5 Survival from relapse according to rituximab treatment at diagnosis or relapse. $C T \rightarrow R-C T$ chemotherapy at diagnosis and immunochemotherapy at relapse; $R-C T \rightarrow R-C T$ immunochemotherapy

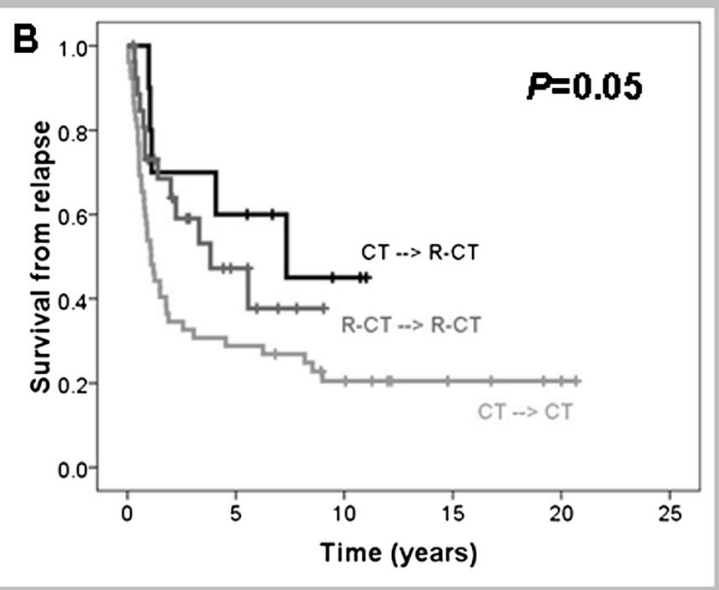

both at diagnosis and at relapse; $C T \rightarrow C T$ chemotherapy both at diagnosis and at relapse; a all cohort; $\mathbf{b}$ only patients treated with curative intention at relapse 
pre-R era with overall response rates between 39 and $69 \%$ including CR rates of 18-48 \% [27-30]. However, in those series, the proportion of primary refractory patients was very low. In fact, our results in the $\mathrm{R}$ era are similar to those reported in recent studies with an overall response of $23-33 \%$ and a CR of $6-8 \%[11,31]$. These differences could be related to the definition of primary refractory disease and the selection of patients. Thus, some series included patients in PR and refractory as a whole. Our definition of refractory disease was strict and included only patients with stable or progressive disease. In summary, in our study, less than $10 \%$ of the refractory patients responded to salvage treatment and were candidate to an eventual intensification. Therefore, it is clear that all refractory patients should be considered for clinical trials with new drugs and novel mechanisms of action.

In contrast to other studies, patients in PR after frontline treatment were grouped separately. The definition of PR can be a problematic one. Nowadays, thanks to PET scan, it is easier to define $\mathrm{PR}$ and to distinguish this situation from $\mathrm{CRu}$ [19]. In our series, the number of patients in PR could have been overestimated before the PET scan availability. Since then, patients with residual masses were considered in PR when PET scan was positive and CR when PET scan was negative. Salvage treatment followed by ASCT is the standard of care for patients in PR. However, this approach is based in old retrospective studies, and there is some controversy since prospective trial data supporting the use of ASCT is lacking, particularly in the R-CT era $[13,14,23,32]$. In the current study, PR patients represented $7 \%$ of all series and showed a considerably better response to salvage treatment and outcome than primary refractory patients. When comparing data between the pre- $\mathrm{R}$ and $\mathrm{R}$ era, a slight improvement in $\mathrm{CR}$ rate and outcome after rescue did not reach statistical significance probably due to the low number of patients. In addition, a higher proportion of patients in this category may benefit from ASCT $[15,16,25,31,33-36]$. In this regard, it is of note that 15 of the 75 patients $(20 \%)$ could receive only palliative measures due to their older age or poor performance status. Conversely, despite the relatively high CR rate, long-term disease-free survival is rather modest with less than one third of patients being cured [25, 26, 31].

The last category is that of patients relapsing after having achieved CR. In recent studies, data indicates that the addition of rituximab to CT implies a substantial reduction of relapse with rates ranging from 20 to $35 \%[8,21,24]$. We have corroborated this finding with relapsing rates of 36 versus $21 \%$ for patients receiving CT or R-CT, respectively. After the PARMA trial, the standard approach for relapsed patients (if eligible) is salvage therapy followed by ASCT. Fiftythree percent of OS is expected in sensitive patients [10]. The only prospective randomized trial of patients with DLBCL in first relapse or primary refractory in the rituximab era is the CORAL, in which 396 patients were randomized in 2 groups of salvage treatment: R-ICE (rituximab, ifosfamide, carboplatin, etoposide) versus R-DHAP (rituximab, dexamethasone, high-dose citarabine, cisplatinum) followed by ASCT in responders. No difference in outcome between the groups was recorded, and a $10 \%$ improvement in terms of outcome was observed with respect to the pre- $\mathrm{R}$ era $[15$, 34-36]. However, Gisselbrecht et al. published that chemotherapy sensitivity before ASCT positively affected response rates, whereas the use of rituximab in first-line treatment had negative effect in those patients relapsing within 12 months after initial CR [15]. The role of rituximab in the second-line setting in refractory patients or in patients whose lymphoma progressed on R-CHOP is also unclear. Current information about the utility of rituximab as part of salvage treatment comes from retrospective analyses [6]. Unfortunately, it does not seem likely that prospective studies are planned to assess this issue. The addition of rituximab to second-line CT followed by ASCT has significantly improved PFS in patients not previously exposed to rituximab [25]. We have also observed that rituximab-naïve patients responded better to salvage treatment followed by ASCT compared to those patients treated both times with rituximab-containing regimens and to those who never received rituximab. This latter group had the worst prognosis. CR duration has been considered one of the most important prognostic factors for outcome in relapsed patients [37]. Interestingly, in our series, patients relapsing after R-CT showed a longer $\mathrm{CR}$ duration than those relapsing after $\mathrm{CT}$ ( 1.5 vs 0.7 years). This could be in part the reason why the short duration of $\mathrm{CR}$ had a modest impact on survival in the present series.

Based on the PARMA trial, ASCT is the standard of care in $\mathrm{R} / \mathrm{R}$ patients with DLBCL responding to salvage treatment. However, no other randomized information is available, particularly in the R era. We must be cautious with results from clinical trials as from other published data concerning salvage treatment approaches since there are most often based on highly selected series of patients in whom intensive treatment is possible $[4,6,10-12,21-25]$. In fact, the median age of those series is low. Most likely such patients who fail initial therapy are not representative of the entire population. In the present series which includes the whole population of $\mathrm{R} / \mathrm{R}$ patients, it is of note that only a minority of those could benefit from intensified treatments. Therefore, the proportion of cases transplanted was 22, 23, and $9 \%$ for relapsed, PR, and refractory patients, respectively. Moreover, the use of rituximab does not seem to have changed this situation.

Prognosis of DLBCL has considerably improved during the last 10 years. However, an important proportion of patients are not yet cured, and the treatment of relapses remains unsatisfactory as shown in the present series. Achieving a sustained CR after first-line therapy is an essential goal. A better biological understanding of early relapses and primarily 
refractory lymphomas is needed, as well as the development of new drugs with different mechanisms of action for $\mathrm{R} / \mathrm{R}$ patients.

Conflict of interest The authors declare that they have no conflicts of interest relevant to the manuscript submission.

Author contributions JR and ALG structured the manuscript. JR, XS, SR AMT, EG, IV LM, and AG collected the relevant data. AV, LC, DM, $\mathrm{AM}$, and EC contributed to the pathology section. JR and ALG wrote the manuscript.

Open Access This article is distributed under the terms of the Creative Commons Attribution License which permits any use, distribution, and reproduction in any medium, provided the original author(s) and the source are credited.

\section{References}

1. The Non-Hodgkin's Lymphoma Classification Project. A clinical evaluation of the International Lymphoma Study Group classification of non-Hodgkin's lymphoma (1997) Blood 89:3909-3918

2. DeVita VT Jr, Canellos GP, Chabner B et al (1975) Advanced diffuse histiocytic lymphoma, a potentially curable disease. Lancet $1: 248-250$

3. Coiffier B, Lepage E, Briere J et al (2002) CHOP chemotherapy plus rituximab compared with $\mathrm{CHOP}$ alone in elderly patients with diffuse large-B-cell lymphoma. N Engl J Med 346:235-242

4. Feugier P, Van Hoof A, Sebban C et al (2005) Long-term results of the R-CHOP study in the treatment of elderly patients with diffuse large B-cell lymphoma: a study by the Groupe d'Etude des Lymphomes de l'Adulte. J Clin Oncol 23:4117-4126

5. Habermann TM, Weller EA, Morrison VA et al (2006) RituximabCHOP versus CHOP alone or with maintenance rituximab in older patients with diffuse large B-cell lymphoma. J Clin Oncol 24:3121-3127

6. Pfreundschuh M, Trümper L, Osterborg A et al (2006) CHOP-like chemotherapy plus rituximab versus CHOP-like chemotherapy alone in young patients with good-prognosis diffuse large-B-cell lymphoma: a randomised controlled trial by the MabThera International Trial (MInT) Group. Lancet Oncol 7:379-391

7. Pfreundschuh M, Schubert J, Ziepert M et al (2008) Six versus eight cycles of bi-weekly CHOP-14 with or without rituximab in elderly patients with aggressive $\mathrm{CD} 20+\mathrm{B}$-cell lymphomas: a randomised controlled trial (RICOVER-60). Lancet Oncol 9:105-116

8. Pfreundschuh M, Kuhnt E, Trümper L et al (2011) CHOP-like chemotherapy with or without rituximab in young patients with good-prognosis diffuse large-B-cell lymphoma: 6-year results of an open-label randomised study of the MabThera International Trial (MInT) Group. Lancet Oncol 12:1013-1022

9. Sehn LH, Donaldson J, Chhanabhai M et al (2005) Introduction of combined CHOP plus rituximab therapy dramatically improved outcome of diffuse large B-cell lymphoma in British Columbia. J Clin Oncol 23:5027-5033

10. Philip T, Guglielmi C, Hagenbeek A et al (1995) Autologous bone marrow transplantation as compared with salvage chemotherapy in relapses of chemotherapy-sensitive non-Hodgkin's lymphoma. $\mathrm{N}$ Engl J Med 333:1540-1545

11. Martín A, Conde E, Arnan M et al (2008) R-ESHAP as salvage therapy for patients with relapsed or refractory diffuse large B-cell lymphoma: the influence of prior exposure to rituximab on outcome. A GEL/TAMO study. Haematologica 93:1829-1836
12. Gutierrez M, Chabner BA, Pearson D et al (2000) Role of a doxorubicin-containing regimen in relapsed and resistant lymphomas: an 8-year follow-up study of EPOCH. J Clin Oncol 18:3633-3642

13. Kewalramani T, Zelenetz AD, Hedrick EE et al (2000) High-dose chemoradiotherapy and autologous stem cell transplantation for patients with primary refractory aggressive non-Hodgkin lymphoma: an intention-to-treat analysis. Blood 96:2399-2404

14. Prince HM, Imrie K, Crump M et al (1996) The role of intensive therapy and autologous blood and marrow transplantation for chemotherapy-sensitive relapsed and primary refractory nonHodgkin's lymphoma: identification of major prognostic groups. $\mathrm{Br}$ J Haematol 92:880-889

15. Gisselbrecht C, Glass B, Mounier N et al (2010) Salvage regimens with autologous transplantation for relapsed large B-cell lymphoma in the rituximab era. J Clin Oncol 28:4184-4190

16. Chen Y-B, Hochberg EP, Feng Y et al (2010) Characteristics and outcomes after autologous stem cell transplant for patients with relapsed or refractory diffuse large B-cell lymphoma who failed initial rituximab, cyclophosphamide, adriamycin, vincristine, and prednisone therapy compared to patients who failed cyclophosphamide, adriamycin, vincristine, and prednisone. Leuk Lymphoma 51:789-796

17. Swerdllow SH, Campo E, Harris NL et al. (2008) WHO classification of tumours of haematopoietic and lymphoid tissues. 4th ed. Lyon, France

18. The International Non-Hodgkin's Lymphoma Prognostic Factors Project. A predictive model for aggressive non-Hodgkin's lymphoma (1993) N. Engl. J. Med. 329:987-994

19. Cheson BD, Pfistner B, Juweid ME et al (2007) Revised response criteria for malignant lymphoma. J Clin Oncol 25:579-586

20. Cox DR (1972) Regression Models and Life-Tables. J R Stat Soc Ser B Methodol 34:187-220

21. Fitoussi O, Belhadj K, Mounier N et al (2011) Survival impact of rituximab combined with ACVBP and upfront consolidation autotransplantation in high-risk diffuse large B-cell lymphoma for GELA. Haematologica 96:1136-1143

22. Gisselbrecht C, Schmitz N, Mounier N et al (2012) Rituximab maintenance therapy after autologous stem-cell transplantation in patients with relapsed CD20(+) diffuse large B-cell lymphoma: final analysis of the collaborative trial in relapsed aggressive lymphoma. J Clin Oncol 30:4462-4469

23. Martelli M, Vignetti M, Zinzani PL et al (1996) High-dose chemotherapy followed by autologous bone marrow transplantation versus dexamethasone, cisplatin, and cytarabine in aggressive non-Hodgkin's lymphoma with partial response to front-line chemotherapy: a prospective randomized italian multicenter study. J Clin Oncol 14:534-542

24. Tarella C, Zanni M, Di Nicola M et al (2007) Prolonged survival in poor-risk diffuse large B-cell lymphoma following front-line treatment with rituximab-supplemented, early-intensified chemotherapy with multiple autologous hematopoietic stem cell support: a multicenter study by GITIL (Gruppo Italiano Terapie Innovative nei Linfomi). Leukemia 21:1802-1811

25. Vellenga E, van Putten WLJ, van 't Veer MB et al (2008) Rituximab improves the treatment results of DHAP-VIM-DHAP and ASCT in relapsed/progressive aggressive CD20+ NHL: a prospective randomized HOVON trial. Blood 111:537-543

26. Villela L, López-Guillermo A, Montoto S et al (2001) Prognostic features and outcome in patients with diffuse large B-cell lymphoma who do not achieve a complete response to first-line regimens. Cancer 91:1557-1562

27. Cabanillas F, Hagemeister FB, Bodey GP, Freireich EJ (1982) IMVP16: an effective regimen for patients with lymphoma who have relapsed after initial combination chemotherapy. Blood 60:693-697

28. Cabanillas F, Hagemeister FB, McLaughlin P et al (1987) Results of MIME salvage regimen for recurrent or refractory lymphoma. J Clin Oncol 5:407-412

29. Velasquez WS, Cabanillas F, Salvador P et al (1988) Effective salvage therapy for lymphoma with cisplatin in combination with high-dose Ara-C and dexamethasone (DHAP). Blood 71:117-122 
30. Velasquez WS, McLaughlin P, Tucker S et al (1994) ESHAP-an effective chemotherapy regimen in refractory and relapsing lymphoma: a 4-year follow-up study. J Clin Oncol 12:1169-1176

31. Telio D, Fernandes K, Ma C et al (2012) Salvage chemotherapy and autologous stem cell transplant in primary refractory diffuse large Bcell lymphoma: outcomes and prognostic factors. Leuk Lymphoma 53:836-841

32. Vose JM (1998) High-dose chemotherapy and hematopoietic stem cell transplantation for relapsed or refractory diffuse large-cell non-Hodgkin's lymphoma. Ann Oncol 9(Suppl 1): S1-S3

33. Kewalramani T, Zelenetz AD, Nimer SD et al (2004) Rituximab and ICE as second-line therapy before autologous stem cell transplantation for relapsed or primary refractory diffuse large B-cell lymphoma. Blood 103:3684-3688
34. Glass B, Ziepert M, Reiser M et al (2010) High-dose therapy followed by autologous stem-cell transplantation with and without rituximab for primary treatment of high-risk diffuse large B-cell lymphoma. Ann Oncol 21:2255-2261

35. Mounier N, Canals C, Gisselbrecht C et al (2012) High-dose therapy and autologous stem cell transplantation in first relapse for diffuse large B cell lymphoma in the rituximab era: an analysis based on data from the European Blood and Marrow Transplantation Registry. Biol Blood Marrow Transplant 18:788-793

36. Mounier N, Gisselbrecht C (2011) Stem cell transplantation for diffuse large B-cell lymphoma patients in the rituximab era. Curr Opin Oncol 23:209-213

37. Guglielmi C, Gomez F, Philip T et al (1998) Time to relapse has prognostic value in patients with aggressive lymphoma enrolled onto the Parma trial. J Clin Oncol 16:3264-3269 